\title{
Frecuencia y tiempo promedio para la rehabilitación postendodontica en una Clinica Dental Docente Peruana
}

\author{
Frecuency and average time for the postendodontic rehabilitation in a Peruvian assistant dental clinic \\ Gaby Oliveira-Ruiz ${ }^{1, a}$, Norka Giuliana Machicao-Chacon 1,a,b, Juan Felipe Hernández-Añaños ${ }^{1, a, b, c}$
}

\section{RESUMEN}

Objetivo: Determinar la frecuencia del tipo de rehabilitación y tiempo promedio que pasa desde la obturación de los conductos radiculares y la rehabilitación postendodóntica en la clínica dental docente de la Facultad de Estomatologia de la Universidad Peruana Cayetano Heredia en el año 2012. Materiales y Métodos: Se revisaron historias clínicas de pacientes atendidos en el Pregrado y en el Postgrado en el año 2012. Muchas historias clínicas fueron excluidas, debido a que no especificaba datos ni en la hoja de evolución ni en la hoja de evaluación. La muestra mínima requerida en la presente investigación fue de 100 tratamientos de conductos para el Pregrado y de 215 para el Postgrado. Resultados: Las molares (51,35\%) fueron las piezas dentarias más frecuentemente tratadas endodonticamente en el Pregrado. En el posgrado la mayor frecuencia también fue para molares $(43,53 \%)$. Los postes de fibra de vidrio fueron la rehabilitación más frecuente: $54 \%$ en el Pregrado y 53,96\% en el Postgrado. El tiempo promedio de rehabilitación postendodóntica en el Pregrado fue 1-14 días, mientras que en el Postgrado el tiempo fue $\geq 45$ días. Conclusiones: En el Pregrado y en el Postgrado, el tratamiento de conductos se realizó con más frecuencia en las molares. El poste de fibra de vidrio fue la rehabilitación postendódontica más frecuente para ambos grupos. El tiempo promedio para rehabilitar las piezas dentarias fue entre 1-14 días en el Pregrado mientras que en el Postgrado lo más frecuente fue $\geq 45$ días.

PALABRAS CLAVE: Microfiltración, tratamiento de conductos, rehabilitación postendodóntica, éxito y fracaso endodóntico. 


\section{SUMMARY}

Objetive: To determine the frequency and time average rate for the postendodntic rehabilitation in the Dental School of Cayetano Heredia Faculty in 2012. Materials and Methods: Medical records of patients treated at the Undergraduate and Graduate in 2012 were review. Many medical records were excluded because they did not specify data sheet or evolution or the evaluation sheet were reviewed. The minimum required sample in this research was 100 root canals for Undergraduate and 215 for Postgraduate. Results: Molars teeth were the most frequently treated in the Undergraduate $(51.35 \%)$ and $(43.53 \%)$ in the postgraduate. Fiberglass posts were the most frequent rehabilitation $54 \%$ in the undergraduate and $53,96 \%$ in the postgraduate. The average time of rehabilitation was $1-14$ days in the Undergraduate, while the time in the postgraduate was $\geq 45$ days. Conclusions: In the undergraduate and postgraduate programs. The root canal treatment was performed more frequently in the molars. The fiberglass post was the most common for both groups. The average time to rehabilitation was between 1-14 days in the undergraduate program while the most frequent postgraduate was $\geq 45$ days.

\section{KEYWORDS: microleakege, root canals, rehabilitation postendodontic, endodontic success and failure.}

\section{INTRODUCCIÓN}

El tratamiento de conductos tiene como objetivo eliminar la infección y prevenir el desarrollo bacteriano en el conducto radicular y los túbulos dentinarios. Este tratamiento juega un papel muy importante en la odontología, ya que el resultado obtenido va influir en los futuros tratamientos, lo cual podría conducir al éxito o fracaso del tratamiento, el cual dependerá del tiempo que se tarde en rehabilitar la pieza dentaria para evitar la microfiltración bacteriana a lo largo del conducto radicular.

Algunas investigaciones, refieren que el fracaso del tratamiento de conductos está asociado a diversos factores, entre los cuales, la filtración apical y coronaria ocupan un lugar importante. Si bien no ha sido aún comprobada la verdadera influencia de la filtración apical en los resultados del tratamiento de conductos, se ha demostrado que la filtración coronaria de saliva, bacterias $\mathrm{u}$ otros elementos tóxicos presentes en el medio bucal pueden penetrar a través de rehabilitaciones defectuosas o ausentes y jugar un rol preponderante en los fracasos a largo plazo (1).

Se menciona que una vez finalizado el tratamiento de conductos, se debe realizar buen sellado de la cavidad de acceso y una rehabilitación inmediata que impida la microfiltración coronaria a largo plazo. En algunos casos se puede encontrar la presencia de una lesión apical o la reaparición de lesiones apicales en dientes tratados en los cuales ya se había visualizado la reparación de áreas radiolúcidas preexistentes como respuesta a un tratamiento exitoso (2).

Hoy en día existen diversas posibilidades de tratamientos que brindan función y estética una vez concluido el tratamiento de conductos, lo cual es importante para tener el éxito y cabe recalcar que el tiempo en el que se realiza la rehabilitación postendodóntica es un factor muy importante.

El objetivo de este estudio fue determinar la frecuencia del tipo y tiempo promedio para rehabilitación postendodóntica en la Clínica Dental Docente de la Facultad de Estomatologia de la Universidad Peruana Cayetano Heredia (FE-UPCH) en el año 2012.

\section{MATERIAL Y MÉTODOS}

Este estudio fue de tipo descriptivo, retrospectivo y observacional. De acuerdo a los datos oficiales proporcionados por la Dirección de Administración de la FE - UPCH, la población estuvo conformada por el número de historias clínicas en la que se haya registrado por lo menos un tratamiento de conductos en el Postgrado y en el Pregrado de la Clínica Dental Docente de la FE - UPCH en el año 2012. En el Pregrado se realizaron 884 tratamientos de conductos en 624 pacientes; y en el Postgrado, 1514 en 1116 pacientes.

Se realizó un estudio piloto en donde se tomó aleatoriamente el $10 \%$ del total de historias clínicas del 2012 que contaron por lo menos con un tratamiento de conductos registrado para obtener los valores pro- 
medios (X) y desviación estándar (S), obteniéndose que la muestra mínima requerida en la presente investigación eran 100 tratamientos de conductos para el Pregrado y 215 para el Postgrado. Se excluyeron en total 80 tratamientos de conductos registrados en las historias clínicas observadas, debido a que no especificaba datos ni en la hoja de evolución ni en la hoja de evaluación, además de no contar con la rehabilitación coronal posterior realizada en alguno de los servicios ofrecidos en la Clínica Dental de la FE - UPCH.

En el estudio se incluyeron historias clínicas que contaron con el registro de tratamientos de conductos de piezas dentales permanentes iniciados y concluidos en la Clínica Dental de la FE - UPCH y tratamientos de conductos que hayan sido rehabilitados que en la Clínica Dental de la FE - UPCH en el año 2012, con registro de la fecha y visto bueno del docente.

Se excluyeron historias clínicas que registraron de tratamientos de conductos y rehabilitación postendodóntica pero que no contaron con el visto bueno del Docente e historias clínicas que no contaron con el registro de la rehabilitación postendodóntica.

Se realizó un análisis de las variables: Fecha de término de tratamiento de conductos, tipo y fecha de inicio del tratamiento de rehabilitación de la pieza y fecha de término de dicho tratamiento. Los resultados se presentaron mediante tablas de frecuencia acumulada, mostrando la distribución porcentual considerando el tipo de pieza dentaria tratada y el servicio que realizó la rehabilitación.
Se realizó una evaluación descriptiva de cada tratamiento de conductos realizado en la Clínica Dental Docente de la FE - UPCH, realizando una distribución de la frecuencia individual de:

Tiempo en días desde la fecha de obturación del tratamiento de conductos hasta la fecha de inicio del tratamiento de rehabilitación.

Tiempo en días desde el inicio del tratamiento de rehabilitación hasta su término.

El registro de los tratamientos de conductos fue codificado manejando la confidencialidad y protección de la información.

\section{RESULTADOS}

En la tabla 1 se presenta la distribución de los tratamientos de conductos efectuados en el Pregrado y Postgrado de la Clínica Dental Docente de la FE UPCH en el año 2012. En el Pregrado y en el Postgrado, los molares fueron las piezas más frecuentemente tratadas $(51,35 \%$ y $43,53 \%$ respectivamente), seguido de las piezas anteriores $(25,80 \%$ en el Pregrado y $29,19 \%$ en el Postgrado) y por último las premolares (22,85\% en el Pregrado y $27,28 \%$ en el Postgrado).

En la tabla 2 se observa que la mayoría de piezas con tratamientos de conductos en el Pregrado y en el Postgrado fueron rehabilitadas con postes de fibra de vidrio (54\% y 53,96\% respectivamente). Seguido del poste colado (36\% en el pregrado y $40,93 \%$ en el Postgrado).

En la tabla 3 se relaciona el tiempo promedio en días

TABLA 1. Distribución de los tratamientos de conductos realizados en el pregrado y postgrado de la Clínica Dental Docente Cayetano Heredia en el año 2012.

\begin{tabular}{lllll}
\hline \multicolumn{4}{c}{ NIVEL ACADÉMICO } \\
& \multicolumn{2}{l}{ PREGRADO } & \multicolumn{2}{l}{ POSTGRADO } \\
\hline TIPO DE PIEZAS DENTALES & $\mathrm{N}$ & $\%$ & $\mathrm{~N}$ & $\%$ \\
Anteriores & 228 & $25,80 \%$ & 442 & $29,19 \%$ \\
Premolares & 202 & $22,85 \%$ & 413 & $27,28 \%$ \\
Molares & 454 & $51,35 \%$ & 659 & $43,53 \%$ \\
TOTAL & 884 & $100 \%$ & 1514 & $100 \%$ \\
\hline
\end{tabular}


TABLA 2. Tipo de rehabilitación postendodóntica realizadas en las piezas dentales tratadas en el pregrado y en el postgrado de la Clinica Dental Docente Cayetano Heredia en el 2012

\begin{tabular}{lllll}
\hline & \multicolumn{4}{c}{ NIVEL ACADÉMICO } \\
& PREGRADO & POSTGRADO \\
\hline TRPE & $\mathrm{n}$ & $\%$ & $\mathrm{n}$ & $\%$ \\
Resina & 0 & $0 \%$ & 0 & $0 \%$ \\
Incrustación & 8 & $8 \%$ & 3 & $1,40 \%$ \\
Poste Colado & 36 & $36 \%$ & 88 & $40,93 \%$ \\
Poste de Fibra de vidrio & 54 & $54 \%$ & 116 & $53,96 \%$ \\
Corona & 2 & $2 \%$ & 8 & $3,71 \%$ \\
TOTAL & 100 & $100 \%$ & 215 & $100 \%$ \\
\hline TRPE: Tipo
\end{tabular}

TRPE: Tipo de Rehabilitación Postendodóntica

Tabla 3. Promedio en días para la rehabilitación postendodóntica de las piezas dentales tratadas en el pregrado y en el postgrado de la Clínica Dental Docente Cayetano Heredia en el año 2012.

\begin{tabular}{lllll}
\hline & \multicolumn{4}{c}{ NIVEL ACADÉMICO } \\
& PREGRADO & \multicolumn{2}{l}{ POSTGRADO } \\
\hline PROMEDIO EN DÍAS & $\mathrm{n}$ & $\%$ & $\mathrm{n}$ & $\%$ \\
1-14 DÍAS & 40 & $40 \%$ & 24 & $11,16 \%$ \\
15-30 DÍAS & 30 & $30 \%$ & 49 & $22,80 \%$ \\
31-44 DÍAS & 11 & $11 \%$ & 27 & $12,56 \%$ \\
$\geq 45$ DÍAS & 19 & $19 \%$ & 115 & $53,48 \%$ \\
TOTAL & 100 & $100 \%$ & 215 & $100 \%$ \\
\hline
\end{tabular}

Tabla 4. Tipo de rehabilitación postendodóntica realizadas en las piezas dentales tratadas en el pregrado y en el postgrado de la Clínica Dental Docente Cayetano Heredia en el año 2012; según el tipo de pieza dental tratada.

\begin{tabular}{|c|c|c|c|c|c|c|c|c|c|c|c|c|}
\hline \multicolumn{13}{|c|}{ TIPO DE PIEZA DENTARIA SEGÚN NIVEL ACADÉMICO } \\
\hline & \multicolumn{6}{|c|}{ PREGRADO } & \multicolumn{6}{|c|}{ POSTGRADO } \\
\hline & \multicolumn{2}{|c|}{ ANTERIORES } & \multicolumn{2}{|c|}{ PREMOLARES } & \multicolumn{2}{|c|}{ MOLARES } & \multicolumn{2}{|c|}{ ANTERIORES } & \multicolumn{2}{|c|}{ PREMOLARES } & \multicolumn{2}{|c|}{ MOLARES } \\
\hline TRPE & $\mathrm{n}$ & $\%$ & $\mathrm{n}$ & $\%$ & $\mathrm{n}$ & $\%$ & $\mathrm{n}$ & $\%$ & $\mathrm{n}$ & $\%$ & $\mathrm{n}$ & $\%$ \\
\hline Resina & 0 & $0 \%$ & 0 & $0 \%$ & 0 & $0 \%$ & 0 & $0 \%$ & 0 & $0 \%$ & 0 & $0 \%$ \\
\hline Incrustación & 0 & $0 \%$ & 3 & $10,34 \%$ & 5 & $13,16 \%$ & 0 & $0 \%$ & 0 & $0 \%$ & 3 & $5,36 \%$ \\
\hline Poste Colado & 7 & $21,21 \%$ & 12 & $41,38 \%$ & 17 & $44,73 \%$ & 37 & $36,28 \%$ & 18 & $31,58 \%$ & 34 & $60,71 \%$ \\
\hline Posteprefabricado & 26 & $78,79 \%$ & 12 & $41,38 \%$ & 16 & $42,11 \%$ & 63 & $61,76 \%$ & 36 & $63,16 \%$ & 16 & $28,57 \%$ \\
\hline Corona & 0 & $0 \%$ & 2 & $6,90 \%$ & 0 & $0 \%$ & 2 & $1,96 \%$ & 3 & $5,26 \%$ & 3 & $5,36 \%$ \\
\hline TOTAL & 33 & $100 \%$ & 29 & $100 \%$ & 38 & $100 \%$ & 102 & $100 \%$ & 57 & $100 \%$ & 56 & $100 \%$ \\
\hline
\end{tabular}

TRPE: Tipo de Rehabilitación Postendodóntica

para la rehabilitación postendodóntica en el Pregrado y en el Postgrado, en el que se observa que el tiempo promedio para la rehabilitación postendodóntica en el Pregrado se dió en el rango de 1-14 días (40\%), mien- tras que en el Postgrado, el tiempo promedio para la rehabilitación postendodóntica se encontró en el rango de $\geq 45$ días. 
Tabla 5. Promedio en días para la rehabilitación postendodóntica en las piezas dentales tratadas en el pregrado y en el postgrado de la Clínica Dental Docente Cayetano Heredia en el año 2012; según el tipo de pieza dental tratada

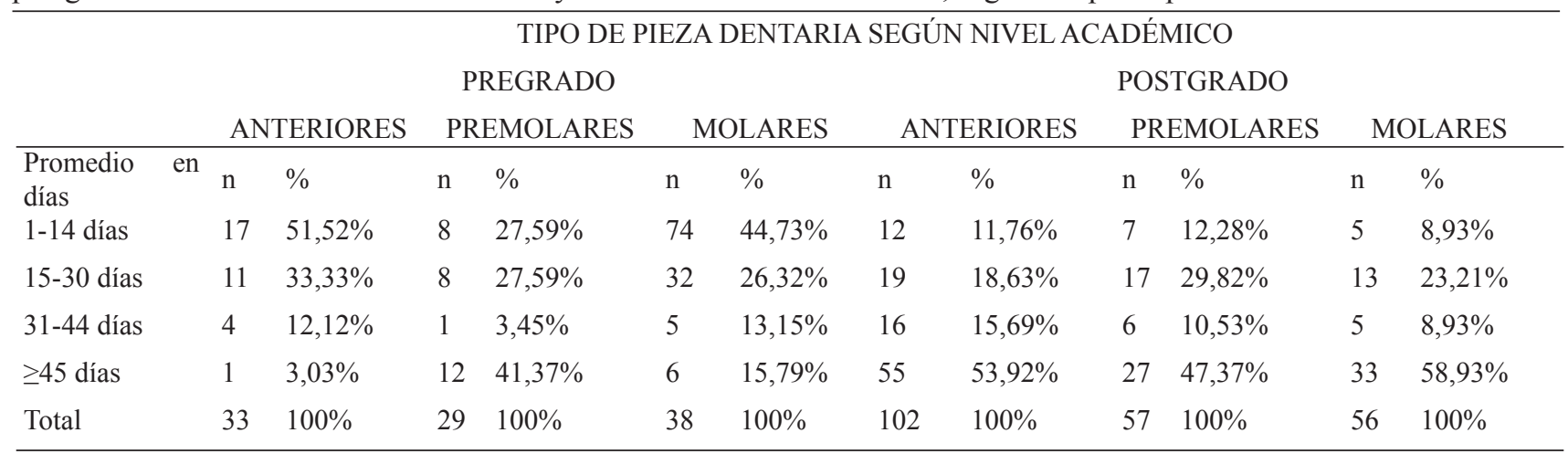

En la tabla 4 se relaciona con la frecuencia del tipo de rehabilitación postendodóntica según el tipo de pieza dental tratada en el Pregrado y en el Postgrado de la Clínica Dental Docente de la FE - UPCH; en el que se encuentra que el poste de fibra de vidrio fue usado mayormente en las piezas anteriores $(78,79 \%)$ en el Pregrado y las premolares $(63,16 \%)$ en el Postgrado. Mientras que las molares fueron rehabilitadas con poste colado (44,73\% en el Pregrado y $60,71 \%$ en el Postgrado).

En la tabla 5 se observa que los dientes anteriores fueron rehabilitadas en un periodo de 1-14 días en el Pregrado $(51,52 \%)$, mientras que en el Postgrado, todos los tipos de dientes fueron rehabilitadas en un rango $\geq 45$ días.

\section{DISCUSIÓN}

La presente investigación tuvo como objetivo determinar la frecuencia del tipo de rehabilitación postendodóntica y tiempo promedio que pasa desde la obturación de los conductos radiculares y el término de la rehabilitación postendodóntica en la Clínica Dental Docente de la FE - UPCH en el año 2012.

Se observó que las molares fueron tratadas con mayor frecuencia en el Pregrado y en el Postgrado (51,35\% y $43,48 \%$ respectivamente). La literatura sobre ello es variada, ya que son solo datos recolectados que no siguen un patrón determinado. Es probable que las molares tengan mayor probabilidad de progresión cariogénica, que pudiese comprometer en algunos casos la pulpa dental. Otras razones pudieran estar relacionadas a tratamientos odontológicos integrales que requieren nivelación del plano de oclusión o tratamiento rehabilitador específico que, buscando una armonía, requiera la desvitalización intencional de algunas piezas dentarias. Salazar, al igual que en el presente estudio, encontró en el Servicio de Endodoncia del Postgrado de la Cínica Dental Docente de la FE - UPCH que las piezas dentarias con mayor frecuencia de necesidad de tratamiento de conductos fueron las molares $(55,85 \%)(3)$. Sin embargo Ingle, quien encontró que las piezas más frecuentemente tratadas endodónticamente fueron los incisivos centrales superiores $(27,1 \%)$. Este basa sus resultados en la tasa de caries, además de los factores estéticos y psicológicos brindados por estas piezas dentales, de ahí el deseo de conservarlas, además de la exposición a traumatismos dentales que ocurre con mayor frecuencia en el sector anterior. También considera que la erupción temprana y afección cariosa hacen que los molares representen también un buen porcentaje de tratamientos de conducto, así mismo relaciona la anatomía que presentan estas piezas dentales, lo que conllevaría a una alta incidencia de lesiones cariosas, debido que los alimentos se ubicaran mayormente en las molares lo que conduce a una necesidad de tratamiento de conductos radícular (4). Por otro lado, investigaciones como las de Sunay y col., señalando que las piezas tratadas con más frecuencia fueron las premolares $(40,2 \%)(5)$ y González y col., donde las piezas anteriores tuvieron mayor frecuencia de tratamientos de conductos $(50,38 \%)(6)$.

Un tratamiento de conductos concluido no debe ser visto como un tratamiento final, sino que la prevención o reparación de la periodontitis apical depende de la rehabilitación final de la pieza tratada (7-10). Tronstad y col., refieren que hay un mayor índice de éxito en el tratamiento de conductos cuando este se 
encuentra asociado con una rehabilitación coronal adecuada (81\%) (11). Ray y Trope evaluaron la relación entre la calidad de la rehabilitación coronaria y la calidad de la obturación del conducto radicular mediante un examen de las radiografias de los dientes tratados endodónticamente. Concluyendo que una combinación de buenas restauraciones y buenos tratamientos mantienen ausente la inflamación periapical en $91,4 \%$ de los casos; mientras que restauraciones pobres y pobres tratamientos de conductos dan como resultado ausencia de inflación periapical en solo $18,1 \%$ de los casos (12). Gilbert y col., explican que el significado de la integridad del sellado coronal ha sido más evidente en el éxito a largo plazo del tratamiento de conductos (10). Machicao y Hernández señalan que cuando la rehabilitación coronal posterior es adecuada, existe 2.85 veces más probabilidad de un éxito en el tratamiento de conductos (13).

Según el grado de destrucción coronaria luego del tratamiento de conductos se decide el tipo de rehabilitación que llevará el diente tratado. Para que el tratamiento de conductos sea preservado es importante el sellado coronal del conducto radicular. Un buen sellado a nivel del conducto radicular previene la microfiltración. De estas la mayoría son rehabilitadas con postes y/o coronas. La colocación adecuada de un poste bien adaptado no es suficiente para asegurar el sellado coronal a largo plazo. En la Clínica Dental Docente de la FE - UPCH las áreas de postgrado de Rehabilitacion Oral y Odontologia Restauradora y Estetica aplican sus propios criterios para la rehabilitación postendodóntica según las nuevas tendencias en las técnicas y materiales, pero aún no está bien establecido ni existe un protocolo aprobado por la FEUPCH de cómo establecer la rehabilitación postendodóntica. En la presente investigación, más del 50\% de casos tratados tanto en el Pregrado, como en el Postgrado se rehabilitaron colocando un poste de fibra de vidrio. Bertorini refiere que los postes prefabricados son muy recomendables, principalmente los postes de fibra de vidrio(14). Esta elección puede deberse a que permiten la preservación de la dentina, contribuyen a una mejor distribución de las fuerzas oclusales a lo largo del eje del diente (15). El módulo de elasticidad es cercano al de la dentina, reduciendo así el número de fracturas radiculares (40 GPa), además son translúcidos lo que colabora con el factor estético (16). Correa y col., comparan 3 tipos de postes prefabricados: fibra de carbono, de vidrio y cerámicos; en cuan- to a la resistencia a la fractura, módulo de elasticidad y propiedades estéticas; obteniendo que los postes de fibra de vidrio muestran mejores resultados (17).

En la presente investigación, también se encontraron altos porcentajes de piezas rehabilitadas con postes colados ( $36 \%$ en el Pregrado y $40,93 \%$ en el Postgrado). Esto podría deberse a que las piezas tratadas endodónticamente no tuvieron suficiente estructura coronaria para ser rehabilitadas de otra manera. Aunque, Torabi y Fattahi el 2009, y Saatian el 2006 comparan la resistencia a la fractura de postes prefabricados y postes colados, concluyendo en ambos estudios que estos últimos presentan mayores porcentajes de fractura radicular por su alto módulo de elasticidad, por lo que podrían ser no recomendables $(18,19)$. Como una alternativa de tratamiento, se utiliza en la rehabilitación los postes anatomisados, que son postes prefabricados de fibra traslucida con confección directa de muñones de resina, endocrown e incrustaciones; endocrown e incrustaciones; lo que podría reemplazar la colocación de postes colados. En la Clínica Dental Docente de la FE - UPCH, estos procedimientos son realizados mayormente en el área de Posgrado en Rehabilitación u Odontología Restauradora y Estética. Bazán y col., refieren que la resistencia de postes elaborados con resina compuesta presenta adecuada resistencia a la compresión.

Para minimizar el riesgo de filtración bacteriana por contacto salival, es esencial que los microorganismos se mantengan alejados de las piezas con tratamiento de conductos (20). Existen muchos materiales para sellar coronalmente de manera provisional las piezas tratadas endodónticamente, entre los que encontramos al ionómeros de vidrio (21), usado en la Clínica Dental Docente de la FE - UPCH. Barthel y col., en 1999 y Khayat y col., en 1993 demostraron en base a las pruebas de microfiltración bacteriana, que en menos de 30 días, las bacterias presentes en la saliva humana pueden penetrar desde las porciones coronales hacia apical, en la totalidad del conducto $(22,23)$. Razón por la cual, se recomienda a los pacientes atendidos en la Clínica Dental Docente de la FE- UPCH, que las piezas con tratamientos de conductos sean rehabilitadas en un periodo no mayor a 30 días.

Lo que llama la atención al evaluar los resultados, es que en el Pregrado las piezas fueron rehabilitadas entre 1-14 días, lo que podríamos considerar favorable 
para el pronóstico de la misma a largo plazo. En el Postgrado, la rehabilitación fue realizada en un periodo de tiempo mayor a lo recomendado, lo que influiría en el éxito de la misma debido a la microfiltración producida. Cabe resaltar, que en el Postgrado, a pesar del tiempo transcurrido, las piezas son rehabilitadas ( $\geq 45$ días) no tomando en cuenta que la microfiltración puede estar presente y conllevar al fracaso. Los estudios refieren que la microfiltración se da a partir de los 30 días.

Las razones que podrían explicar la demora del tratamiento de rehabilitación puede radicar en la falta de información proporcionada al paciente y/o la conciencia de continuar el tratamiento integral, ya que algunos pacientes que acuden al Postgrado sólo toman importancia al motivo de dolor y una vez solucionado no continúan con el tratamiento integral. Otra limitante podría ser el factor económico, en el Pregrado la mayoría de veces es el operador quien asume los gastos del tratamiento, lo que podría comprometer al paciente en acudir a sus citas en un periodo de tiempo menor; mientras que en el Postgrado, es el paciente quien asume el costo del tratamiento, lo que podría limitar a cumplir con la rehabilitación integral programada. Uno de los factores que también podrían influir es la disponibilidad de las citas en los diferentes servicios, a veces la postergación del inicio del tratamiento se da porque el paciente no puede conseguir una cita en el servicio al que fue derivado, por lo que preferiría hacerlo fuera de la clínica por tener citas más próximas. No se puede olvidar que cuando un paciente llega al Servicio para recibir un tratamiento este firma un consentimiento informado para proceder a la ejecución del tratamiento, en el cual el acepta las indicaciones, contraindicaciones y posibles consecuencias. También se debe tomar en cuenta, que los tratamientos rehabilitadores en la Clínica Dental Docente de la FE - UPCH son realizados en diferentes servicios y muchas veces no son vistos de manera integral.

El presente estudio ha permitido determinar la frecuencia del tipo y tiempo promedio para rehabilitación postendodóntica de dientes tratados endodónticamente en la Clínica Dental Docente de la FE - UPCH en el año 2012. Sin embargo, es necesario resaltar la importancia de realizar múltiples estudios que complementen los resultados obtenidos en la presente investigación.

\section{CONCLUSIONES}

Las molares fueron los dientes más frecuentemente tratados en el Pregrado y en el Postgrado de la Clínica Dental Docente de la FE - UPCH en el año 2012.

El poste de fibra de vidrio fue la rehabilitación postendóndotica más frecuente en el Pregrado y en el Postgrado de la Clínica Dental Docente de la FE $\mathrm{UPCH}$.

El promedio para rehabilitar piezas dentales con tratamiento de conductos fue entre 1-14 días en el Pregrado mientras que en el Postgrado fue más frecuente en $\geq 45$ días.

Las piezas anteriores fueron rehabilitadas frecuentemente con postes de fibra de vidrio $(78,79 \%)$, seguido de las molares con poste colado $(44,73 \%)$ en el Pregrado. Mientras que en el Postgrado, las premolares fueron rehabilitadas con poste de fibra de vidrio $(63,16 \%)$ y las molares con poste colado $(60,71 \%)$.

Las piezas anteriores fueron rehabilitadas en un periodo de 1-14 días en el Pregrado (51,52\%), mientras que en el Postgrado, las molares fueron rehabilitadas en un rango $\geq 45$ días $(58,93 \%)$.

\section{Correspondencia:}

Gaby Oliveira Ruiz

Correo electrónico: gaby.oliveira@upch.pe

\section{REFERENCIAS BIBLIOGRÁFÍCAS}

1. Zmener O. Mejorando el sellado coronario en endodoncia. Endodoncia (Madrid). 2009; 27, (4): 201209.

2. Barrientos P. Contaminación post-endodóntica vía coronaria: Un frecuente factor de fracaso. Rev Dent Chile. 2003; 94(2): 35-6.

3. Salazar L. Determinación de la frecuencia de las piezas dentarias permanentes con mayor necesidad de tratamiento de conductos radicular en pacientes que acudieron al servicio de Postgrado de Tratamiento de conductos de la Clínica Estomatológica Central de la Universidad Peruana Cayetano Heredia, durante el periodo Enero 1995 a Enero 1997. Tesis de Bachiller Lima, Perú: Universidad Peruana Cayetano Heredia; 1999. 
4. Ingle JI, Taintor JF. Endodontics. 3rd. Ed. Philadelphia: Leas Febiger, 1985.p. 34-5.

5. Sunay H, Tanalp J, Dikbas I, Bayirli G. Cross-sectional evaluation of the periapical status and quality of root canal treatment in a selected population of urban Turkish adults. Int Endod J. 2007; 40: 139-45.

6. Gonzáles A, Quintana M, Matta C, Maldonado M. Frecuencia de diagnósticos y tratamientos pulpares según indicadores de la demanda realizados en una clínica dental universitaria. Rev Estomatol Herediana. $2005 ; 15$ (2): 150-4.

7. Bergenholtz G, Horsted-Bindslev P, Reit CI. Tratamiento de conductos: Diagnóstico y tratamiento de la pulpa dental. Ciudad de México: El Manual Moderno; 2007.

8. Dillard Ch, Barfield R, Tilashalski K, Chavers L, Eleazar P. Comparison of endodontist versus generalist regarding preference for postendodontic use of cotton pellets in pulp chamber. J Endod. 2002; 28(9):656-7.

9. Endodontics Colleagues for Excellence. Microleakage coronal. AAE 2005; 23(3): 201-6.

10. Gilbert SD, Witherspoon DE, Berry CW. Coronal leakage following three obturation techniques. Int Endod J 2001; 34: 293-9.

11. Tronstad L, Asbjørnsen K, Døving L. Pedersen I, Eriksen H. Influence of coronal restorations on the periapical health of endodontically treated teeth. Dent traumatol. 2000; 16(5): 218-21.

12. Ray HA, Trope M. Periapical status of endodontically treated teeth in relation to the technical quality of the root filling and the coronal restoration. Int Endod J. 1995; 28(1): 12-8.

13. Machicao N, Hernández JF. Estado clínico y radiográfico de tratamientos endodóncicos en pacientes del Servicio de Endodoncia del Postgrado de la Facultad de Estomatología de la Universidad Peruana Cayetano Heredia en el año 2012. Tesis de Maestría Lima, Perú: Universidad Peruana Cayetano Heredia; 2015.

14. Bertorini C. Resistencia a la tracción en espigos de fibra de vidrio cementados con un cemento a base de resina y otro a base de ionómero de vidrio. Tesis de Cirujano Dentista. Lima, Perú: Universidad de San Martin de Porres; 2009.

15. Farina A, Cecchin D, Da Fonseca Roberti L, Zago L, De Carvalho Panzeri F. Bond strength of fibre glass and carbon fibre posts to the root canal walls using different resin cements Aust Endod J. 2011; 37: 4450.

16. Ramirez-Ortega J, García M, Magaña C. Caracterización de postes intrarradiculares de fibra de vidrio. Acapulco, Mexico: Octavo Congreso Internacional de Microscopia; 1 al 6 de octubre del 2006.

17. Correa A, Westphalen G, Ccahuana V. Sistema de postes estéticos reforzados. Rev Estomatol Herediana. 2007; 2: 99-103.
18. Torabi K, Fattahi F. Fracture resistance of endodontically treated teeth restored by different FRC posts: An in vitro study. Indian J Dent Res. 2009; 20: 282-7.

19. Saatian S. Fracture strength of endodontically treated teeth restored with casting post and core and glass-fiber with composite core. Iranian Endodontic Journal. 2006; 1: 65-8.

20. Baumann M, Beer R. Tratamiento de conductos. Segunda edición. Madrid: Elsevier Mosby; 2008.

21. Kopper PMP, Figueiredo JAP, Della Bona A, Vanni JR, Bier CA, Boop S. Comparative in vitro analysis of the sealing ability of three endodontic sealers in post-prepared root canals. Int Endod J. 2003; 36: 85763.

22. Barthel CR, Strobach A, Briedigkeit H, Göbel UB, Roulet J-F. Leakage in roots coronally sealed with different temporary fillings. Int Endod J 1999; 25 (11): 731- 4.

23. Khayat A, Lee SJ, Torabinejad M. Human saliva penetration of coronally unsaled obturated root Canals. J Endod. 1993; 19:458-61.

Recibido : 20/01/2016

Aceptado: 12/04/2016 